\title{
The myloglossus in a human cadaver study: common or uncommon anatomical structure?
}

\author{
B. Buffoli*, M. Ferrari*, F. Belotti, D. Lancini, M.A. Cocchi, M. Labanca, M. Tschabitscher, \\ R. Rezzani, L.F. Rodella
}

Section of Anatomy and Physiopathology, Department of Clinical and Experimental Sciences, University of Brescia, Brescia, Italy

[Received: 1 June 2016; Accepted: 18 July 2016]

\begin{abstract}
Background: Additional extrinsic muscles of the tongue are reported in literature and one of them is the myloglossus muscle (MGM). Since MGM is nowadays considered as anatomical variant, the aim of this study is to clarify some open questions by evaluating and describing the myloglossal anatomy (including both MGM and its ligamentous counterpart) during human cadaver dissections.

Materials and methods: Twenty-one regions (including masticator space, sublingual space and adjacent areas) were dissected and the presence and appearance of myloglossus were considered, together with its proximal and distal insertions, vascularisation and innervation.

Results: The myloglossus was present in $61.9 \%$ of cases with muscular, ligamentous or mixed appearance and either bony or muscular insertion. Facial artery provided myloglossal vascularisation in the $84.62 \%$ and lingual artery in the $15.38 \%$; innervation was granted by the trigeminal system (buccal nerve and mylohyoid nerve), sometimes (46.15\%) with hypoglossal component.

Conclusions: These data suggest us to not consider myloglossus as a rare anatomical variant. (Folia Morphol 2017; 76, 1: 74-81)
\end{abstract}

Key words: anatomical variant, dissection, myloglossus, tongue

\section{INTRODUCTION}

The muscular architecture of the tongue is a very complex anatomical system. The muscles are conventionally divided into intrinsic muscles, which arise and insert into the organ determining its shape, and extrinsic muscles, which arise from external structures and insert into the tongue acting on its movements in the oral cavity during mastication, deglutition and phonation.

Four pairs of extrinsic muscles are commonly identified in relation to their location and fibre direction: genioglossus, hyoglossus, styloglossus (SGM), and palatoglossus muscle. However, in view of its peculiar vascularisation, nerve supply, embryogenesis, and preeminent swallowing function, the palatoglossus muscle is sometimes not considered a muscle of the tongue.

Furthermore, additional extrinsic muscles, which are not usually considered in the anatomy textbooks, such as amigdaloglossus $[2,12,16]$, pharyngoglossus [12], chondroglossus, and myloglossus muscle (MGM) have been also described [17].

Myloglossus muscle was at first named by Winslow from "mylo-", that means "mandible", and "-glossus", that means "tongue". It was initially described as a group of additional muscular fibres run-

Address for correspondence: Prof. L.F. Rodella, Section of Anatomy and Physiopathology, Department of Clinical and Experimental Sciences, University of Brescia, V.le Europa 11, 25123, Brescia, Italy, tel: +39 0303717485, fax: +39 0303717486, e-mail: luigi.rodella@unibs.it 
ning from the posterior part of the mandible (near the mylohyoid line) to the side of the tongue [3] or "from the angle of the lower jaw, and also from the stylo-maxillary ligament to the side of the tongue" [5]. Later, Wood [19] considered MGM as part of the superior pharyngeal constrictor muscle reaching the side of the tongue and Macalister $[9,10]$ defined as MGM every group of muscular fibres arising from the stylomandibular ligament, medial pterygoid muscle or mandible and reaching the tongue. In 1926, Valenti [18] used the term "mandibulo-glossus" referring to MGM. More recently, MGM was described to arise from the medial surface of the angle of the mandible, close to the mandibular insertion of medial pterygoid muscle, and reach the SGM and/or hyoglossus muscles [13].

The prevalence of the muscle was reported being $84 \%$ by Lang and Jude [8]. Mérida-Velasco et al. [11] identified a fibrous or muscular structure joining the angle of the mandible or the stylomandibular ligament with the SGM in their entire sample. However, MGM is nowadays considered an anatomical variant in the scientific literature. The aim of this study is to clarify some open questions by evaluating and describing the MGM anatomy during human cadaver dissections.

\section{MATERIALS AND METHODS}

Eleven adult human heads were dissected. The specimens were fixed in phenol-formalin solution and the arteries were injected with red silicone rubber.

Twenty-one regions (including masticatory space, sublingual and submandibular fossae, and adjacent areas) were systemically dissected using two approaches: 1) partial or total resection of the mandibular ramus and analysis of the deeper spaces; 2) en-bloc removal of the mandible with surrounding soft tissues and dissection.

The following features were systematically evaluated:

- Presence and appearance of a myloglossal structure lying between the angle of the mandible/the stylomandibular ligament/the medial pterygoid muscle and the oral tongue or the SGM. About the appearance of the myloglossal structure, we classified it as muscular (i.e. MGM), ligamentous (i.e. myloglossal ligament [MGL]) or mixed, i.e. a MGL with few muscle fibres. We decided to consider this latter together with MGL.

- Size of ipsilateral SGM: normal-sized or small-sized $(<8 \mathrm{~mm})$.
- Distal and proximal insertion of myloglossal structure. The distal insertion was considered "lingual" when the muscle inserted onto the tongue muscles or "styloglossal" when the muscle inserted onto the SGM before it reached the tongue. Proximal insertion was classified as bony (i.e. tuberculum alveolare mandibulae, posterior border of the angle of the mandible, or lower surface of pterygoid tuberosity) or muscular (i.e. medial pterygoid muscle).

- Vascularisation. It was analysed by identifying the main artery supplying myloglossal structure and its branching pattern.

- Innervation. It was analysed by identifying the nerve(s) giving a branch supplying myloglossal structure.

In addition, high-definition endoscopic camera with $0^{\circ} 4 \mathrm{~mm}$ Hopkins Rod-lens scope (KARL STORZ ${ }^{\circledR}$, Tuttlingen, Germany) was adopted to better analyse and identify the anatomical structures without a large dissection.

All these data were arranged in contingency tables and Fisher's exact test was performed.

\section{RESULTS}

Presence and appearance. A muscular, ligamentous or mixed myloglossal structure was observed in $61.90 \%$ (13/21 cases). Among them, MGM was observed in $38.46 \%$ ( $5 / 13$ cases), MGL in $23.08 \%$ (3/13) of cases and mixed in $38.46 \%$ (5/13 cases) (Fig. 1, Table 1).

Size of ipsilateral SGM. A prevalence of normalsized SGM (61.54\%) vs. small-sized SGM (38.46\%) was observed in concomitance with myloglossal structure. In particular, we found a normal-sized SGM in $20 \%(1 / 5)$ of MGM cases and small-sized SGM in $80 \%$ ( $4 / 5$ cases). In addition, we found normal-sized SGM in $87.5 \%(7 / 8)$ of MGL or mixed cases and small-sized SGM in $12.5 \%$ (1/8 cases) (Table 1$)$. This association was statistically significant $(p=0.032$ ).

Distal and proximal insertion. About distal insertion, lingual insertion (into the hyoglossus between the palatoglossal and the styloglossal insertions) was found in $30.77 \%$ (4/13 cases), whereas styloglossal insertion in $69.23 \%$ (9/13 cases). More precisely, lingual insertion was present in $80 \%$ ( $4 / 5$ cases) of MGM whereas it was not found in MGL or mixed myloglossal structure where $100 \%$ of styloglossal insertion was observed (Figs. 2, 3, Table 1). This association was statistically significant $(p=0.007)$. About proximal 

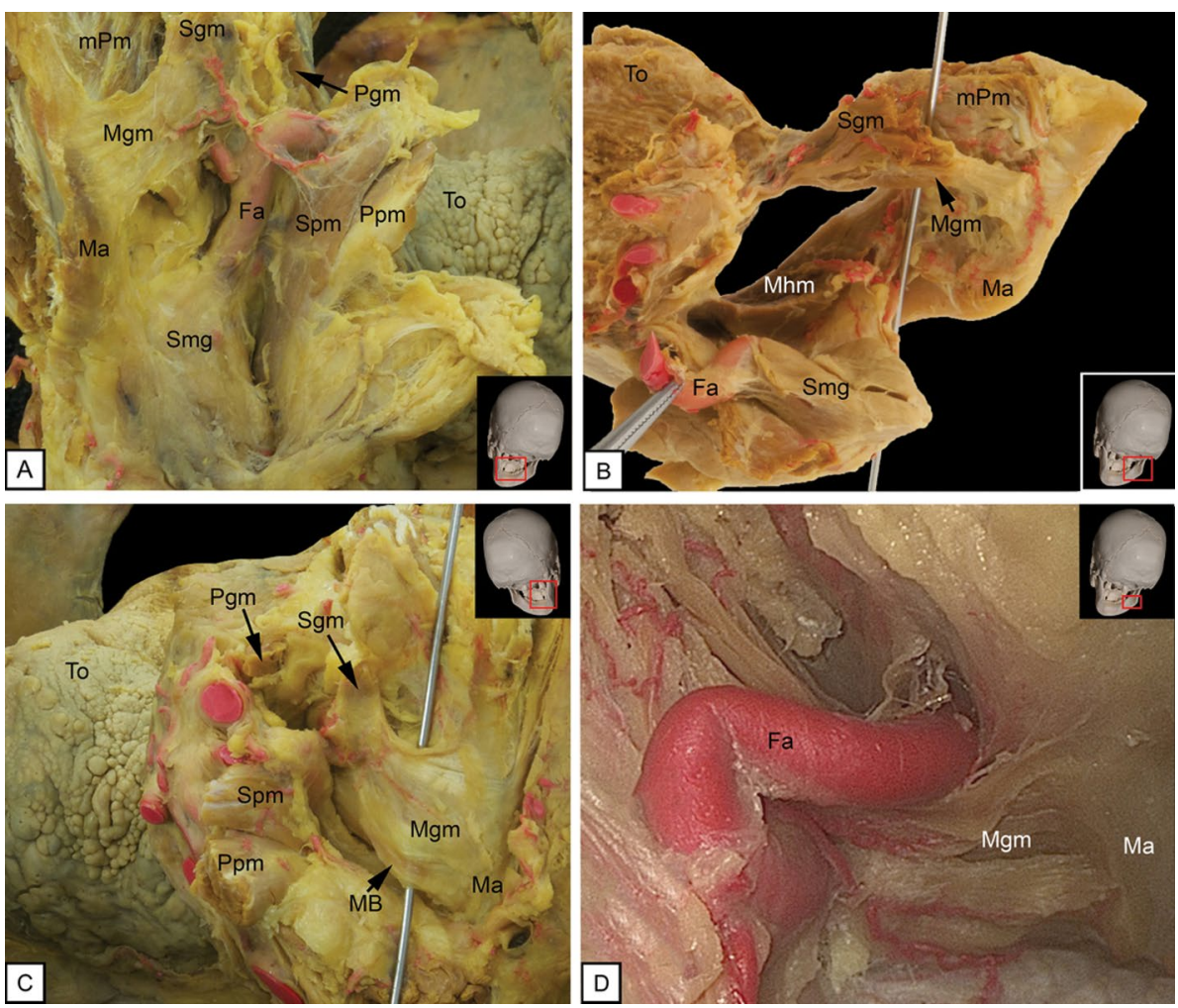

Figure 1. Appearance of myloglossus; A. Ligamentous appearance; B. Mixed appearance: the muscular bundles arise from a ligamentous tract; C. Mixed appearance: the muscular bundles arise from the bone directly; $\mathbf{D}$. Muscular appearance: the muscle was located below the facial artery (posterior-to-anterior endoscopic view); Fa — facial artery; $\mathrm{Ma}$ - mandible angle; MB — muscular bundle; Mgm — myloglossus muscle; Mhm — mylohyoid muscle; mPm — medial pterygoid muscle; Pgm — palatoglossus muscle; Ppm — palatopharyngeus muscle; Sgm — styloglossus muscle; Smg — submandibular gland; Spm — stylopharyngeus muscle; To — tongue.

Table 1. The correlation between myloglossal structure appearance (muscular, ligamentous or mixed), size of ipsilateral styloglossus muscle (SGM), distal insertion and proximal insertion. Percentages in square brackets represent the partial percentages base on the different myloglossal appearance (myloglossus muscle [MGM], $n=5$; myloglossal ligament [MGL], $n=3+$ mixed, $n=5$ ); the percentage values represent the whole percentage $(n=13)$

\begin{tabular}{llcc}
\hline \multicolumn{2}{l}{ Myloglossus appearance - insertion } & MGM (n= 5, 38.46\%) & $\begin{array}{c}\text { MGL (n= 3, 23.08\%) } \\
+ \text { Mixed (n= 5, 38.46\%) }\end{array}$ \\
\hline \multirow{2}{*}{ Ipsilateral SGM } & Normal size: $61.54 \%$ & {$[20 \%] ; 7.69 \%$} & {$[87.5 \%] ; 53.85 \%$} \\
& Small size: $38.46 \%$ & {$[80 \%] ; 30.77 \%$} & {$[12.5 \%] ; 7.69 \%$} \\
\hline Distal insertion & Lingual: $30.77 \%$ & {$[80 \%] ; 30.77 \%$} & {$[0 \%] ; 0 \%$} \\
& SGM: $69.23 \%$ & {$[20 \%] ; 7.69 \%$} & {$[100 \%] ; 61.54 \%$} \\
\hline Proximal insertion & Bony: $76.92 \%$ & {$[40 \%] ; 15.38$} & {$[100 \%] ; 61.54 \%$} \\
& Muscular: $23.08 \%$ & {$[60 \%] ; 23.08$} & {$[0 \%] ; 0 \%$} \\
\hline
\end{tabular}

insertion, we observed a prevalence of bony insertion $(76.92 \%$, i.e. $10 / 13$ cases) vs. muscular insertion (23.08\%, i.e. $3 / 13$ cases). Bony insertion was located on the tuberculum alveolare mandibulae in $15.38 \%$ (2/13 cases), on the posterior border of the angle of the mandible in $23.08 \%$ ( $3 / 13$ cases) or on the lower surface of pterygoid tuberosity in the $38.46 \%(5 / 13$ cases). In particular, bony insertion was found in $40 \%$ (2/5 cases) and muscular insertion in 60\% (3/5 cases) in MGM; in MGL or mixed cases, $100 \%$ of bony insertion was found (Table 1). This association was statistically significant $(p=0.035)$.

Vascularisation. Myloglossus (muscular, tendinous or mixed) was supplied by the external carotid 


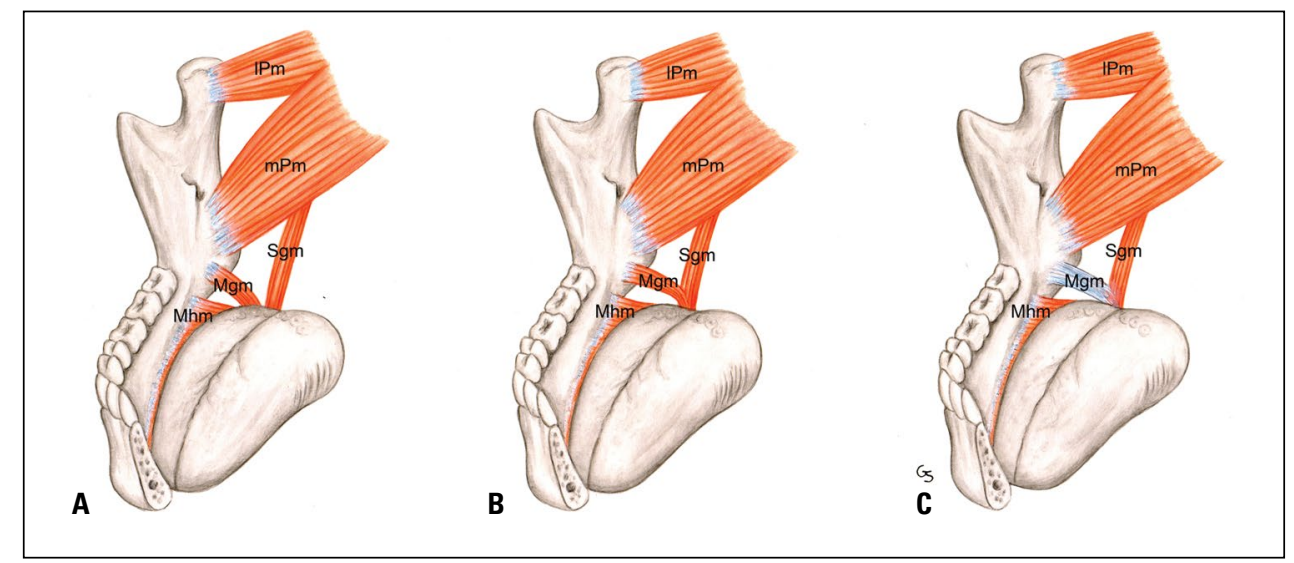

Figure 2. Schematic representation of the appearance and insertion of myloglossus; A. Muscular appearance and lingual insertion; B. Muscular appearance and styloglossal insertion; C. Ligamentous appearance and styloglossal insertion; Mgm — myloglossus muscle; Mhm — mylohyoid muscle; mPm — medial pterygoid muscle; IPm — lateral pterygoid muscle; Sgm — styloglossus muscle.
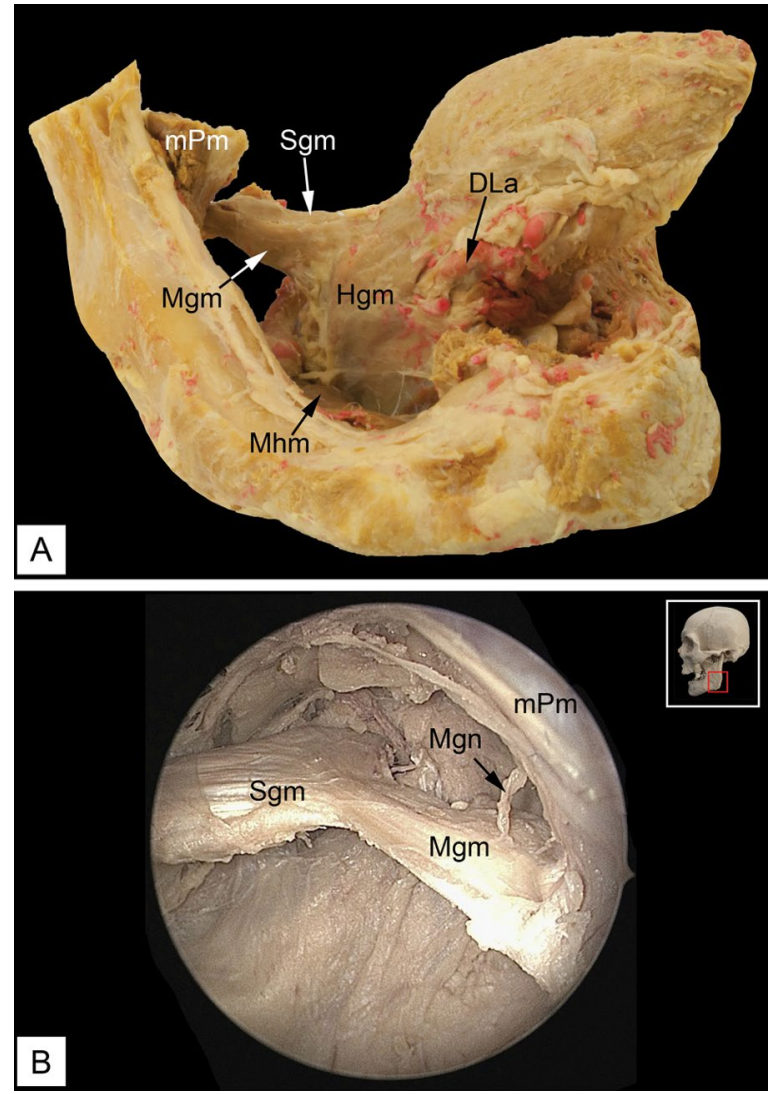

Figure 3. Distal insertion of myloglossus; A. Lingual insertion of the myloglossus muscle reaching a space between the hyoglossus muscle and the styloglossal insertion on the tongue; B. Styloglossal insertion of the mixed myloglossus (lateral-to-medial endoscopic view); DLa deep lingual artery; Hgm — hyoglossus muscle; Mgm — myloglossus muscle; Mgn — myloglossus nerve; Mhm — mylohyoid muscle; mPm — medial pterygoid muscle; Sgm — styloglossus muscle.

artery: from the facial artery in $84.62 \%$ (11/13 cases) and from the lingual artery in $15.38 \%$ (2/13 cases).
Different branching patterns were found:

- Pattern A: presence of single branch entirely distributed to the myloglossus (muscular, ligamentous or mixed);

- Pattern B: presence of single branch distributed both to the myloglossus and to the medial pterygoid muscle;

- Pattern C: presence of two branches, the first corresponding to the pattern $A$ and the second to the pattern $B$;

- Pattern D: presence of single branch distributed both to myloglossus and to the SGM.

The blood supply from the facial artery was slightly equally-distributed in the four patterns: pattern $A$ in $36.36 \%$ (4/11 cases); pattern $B$ in $18.18 \%(2 / 11$ cases); pattern C in $18.18 \%$ ( $2 / 11$ cases); pattern D in $27.27 \%$ ( $3 / 11$ cases). On the other hand, about the lingual artery, we observed an equal distribution of pattern A ( $1 / 2$ cases) and B ( $1 / 2$ cases) (Fig. 4, Table 2 ).

Innervation. Myloglossal innervation was provided by branch(es) arising from the mandibular nerve $\left(V_{3}\right)$ and hypoglossal nerve. The mandibular nerve supplied the muscle through the buccal nerve, releasing a myloglossal branch inferolaterally before entering the buccal space; or the mylohyoid nerve, giving a myloglossal branch while it passed on its lateral side. On the other hand, the hypoglossal nerve released an ascending branch just before passing over the mylohyoid muscle, similarly to the styloglossal innervation. Our data showed that the myloglossal nervous branch(es) arose from the buccal nerve in $69.23 \%$ (9/13 cases) and from the mylohyoid nerve in $30.77 \%$ (4/13 cases). Additional innervation from hypoglossal nerve was found in $44.44 \%$ (4/9 cases) 

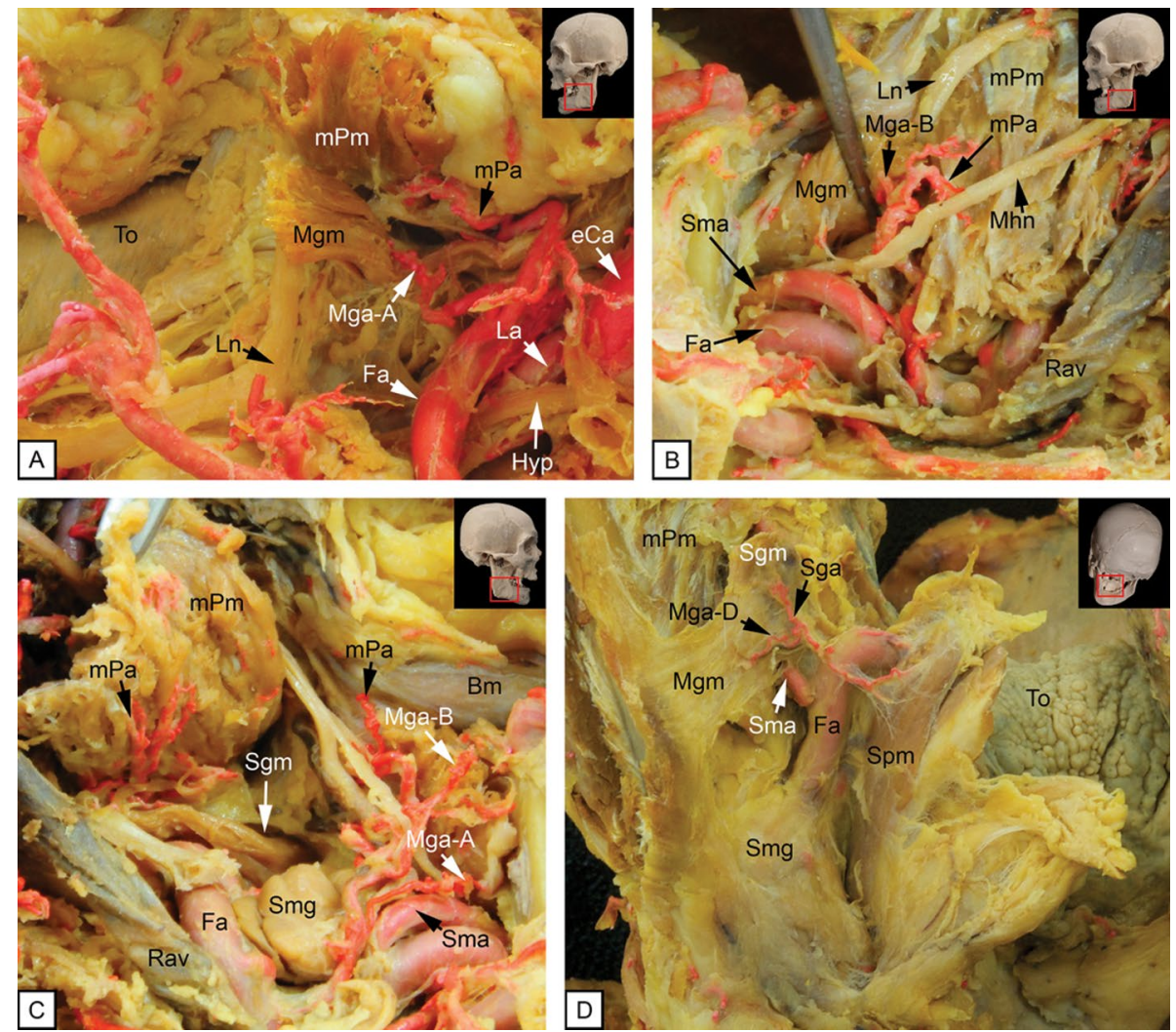

Figure 4. Vascularisation patterns of myloglossus; A. Pattern A (single branch entirely dedicated to the muscle); B. Pattern B (single branch dedicated both to the myloglossus muscle and to the medial pterygoid muscle); C. Pattern $C$ (two branches, pattern $A$ and $B$ ); D. Pattern $D$ (single branch dedicated both to the myloglossus muscle and to the styloglossus muscle); $\mathrm{Bm}$ - buccinator muscle; eCa - external carotid artery; Fa — facial artery; Hyp — hypoglossus nerve; La — lingual artery; Ln — lingual nerve; Mga(-A/-B/-D) — myloglossal artery (pattern $\mathrm{A}, \mathrm{B}$ and $\mathrm{D}$ ); $\mathrm{Mgm}$ — myloglossus muscle; $\mathrm{Mhn}$ — mylohyoid nerve; $\mathrm{mPa}$ - medial pterygoid artery (cut); $\mathrm{mPm}$ — medial pterygoid muscle; Rav — retroangolomandibular vein; Sga — styloglossal artery; Sgm — styloglossus muscle; Sma — submental artery; Smg — submandibular gland; Spm — stylopharyngeus muscle; To — tongue.

Table 2. The vascularisation patters of the myloglossal structure. See the text for further description of the vascularisation patterns (Pattern A-B-C-D). Percentages in square brackets represent the partial percentage for each artery (facial artery, $\mathrm{n}=11$; lingual artery, $n=2)$; the percentage values represent the whole percentage $(n=13)$

\begin{tabular}{lcccc}
\hline Vascularisation & Pattern A & Pattern B & Pattern C & Pattern D \\
\hline Facial artery $(\mathrm{n}=11 ; 84.62 \%)$ & {$[36.36 \%] ; 30.77 \%$} & {$[18.18 \%] ; 15.38 \%$} & {$[18.18 \%] ; 15.38 \%$} & {$[27.27 \%] ; 23.08 \%$} \\
Lingual artery $(\mathrm{n}=2 ; 15.38 \%)$ & {$[50 \%] ; 7.69 \%$} & {$[0 \%] ; 0 \%$} & {$[0 \%] ; 0 \%$} & {$[50 \%] ; 7.69 \%$} \\
\hline
\end{tabular}

with buccal nerve and $50 \%$ (2/4 cases) with mylohyoid nerve. Purely hypoglossal innervation was not found (Fig. 5, Table 3).

Endoscopic overview representing the main topographic relationship of myloglossus was showed in Figure 6.

\section{DISCUSSION}

Several definitions have been previously made for $\operatorname{MGM}[3,5,8,11,18,19]$. It was extremely confus- ing and has led to MGM being considered as a rare anatomical variant [13].

In accordance with the concept of the Virchow's archive [4], a cumulative analysis of MGM including its ligamentous counterpart could be of some use to the purpose of clarifying the anatomy of this muscle; thus, we decided to consider also MGL and mixed structure.

A total of 21 regions were evaluated during this study. We observed MGM, MGL or mixed myloglossus 


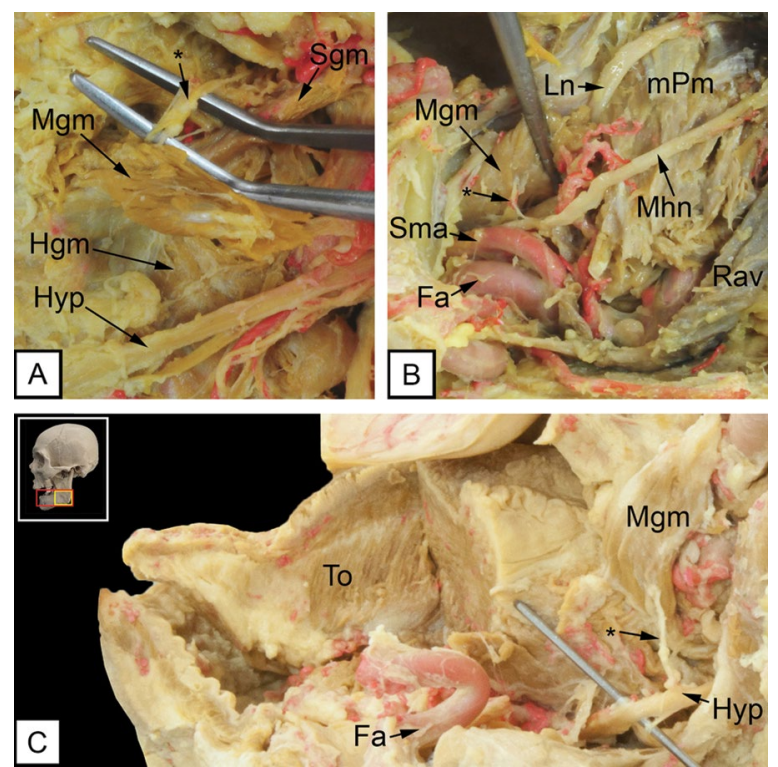

Figure 5. Innervation patterns of myloglossus; A. The myloglossal nerve arises from the buccal nerve; $\boldsymbol{B}$. The myloglossal nerve arises from the mylohyoid nerve; $\mathbf{C}$. The hypoglossal component of innervation for the myloglossus muscle; $\mathrm{Fa}$ - facial artery; $\mathrm{Hgm}-$ hyoglossus muscle; Hyp - hypoglossus nerve; Ln — lingual nerve; Mgm — myloglossus muscle; Mhn — mylohyoid nerve; $\mathrm{mPm}$ - medial pterygoid muscle; Rav — retroangolomandibular vein; Sgm — styloglossus muscle; Sma - submental artery; To - tongue; *Myloglossal nerve.

in $61.90 \%(13 / 21$ cases), similarly to the $84 \%$ rate reported by Lang and Jude [8]. Furthermore, accessory muscular or ligamentous fibres going from the mandible angle to the SGM were also observed by Mérida-Velasco et al. [11] in human foetus. These data supported our idea that these structures are not rare anatomical variants and lead us to give
Table 3. The innervation patterns of the myloglossal structure. Percentages in square brackets represent the partial percentage of additional hypoglossal innervation for each nerve of the trigeminal system (buccal nerve, $\mathrm{n}=9$; mylohyoid nerve, $n=4)$; the percentage values represent the whole percentage ( $n=13$ )

\begin{tabular}{lcc}
\hline Innervation & $\begin{array}{c}\text { With hypoglossal } \\
\text { branch }\end{array}$ & $\begin{array}{c}\text { Without hypoglossal } \\
\text { branch }\end{array}$ \\
\hline $\begin{array}{l}\text { Buccal nerve } \\
(\mathrm{n}=9 ; 69.23 \%)\end{array}$ & {$[44.44 \%] ; 30.77 \%$} & {$[55.55 \%] ; 38.46 \%$} \\
$\begin{array}{l}\text { Mylohyoid nerve } \\
(\mathrm{n}=4 ; 30.77 \%)\end{array}$ & {$[50 \%] ; 15.38 \%$} & {$[50 \%] ; 15.38 \%$} \\
\hline
\end{tabular}

a detailed anatomical description of them based on the following points:

Size of ipsilateral SGM. Our data showed an inverse correlation between the presence of MGM and the size of ipsilateral SGM. These results could be considered in accordance with another work showing that when MGM was found, SGM tends to be small-sized [13].

Distal and proximal insertion. Although each Author reported the proximal insertion as a constant, there is a wide and heterogeneous range of observations in the literature $[3-5,8,13,18]$. Proximal insertion was described as the "posterior part of the mylohyoid line" [3], "the angle of the lower jaw and also from the stylo-maxillary ligament" [5], "the inner surface of the lower jaw" [18], the area of the tuberculum alveolare mandibulae [8] or "the inner surface of the medial pterygoid muscle" $[4,13]$. In this study, different types of proximal insertion were

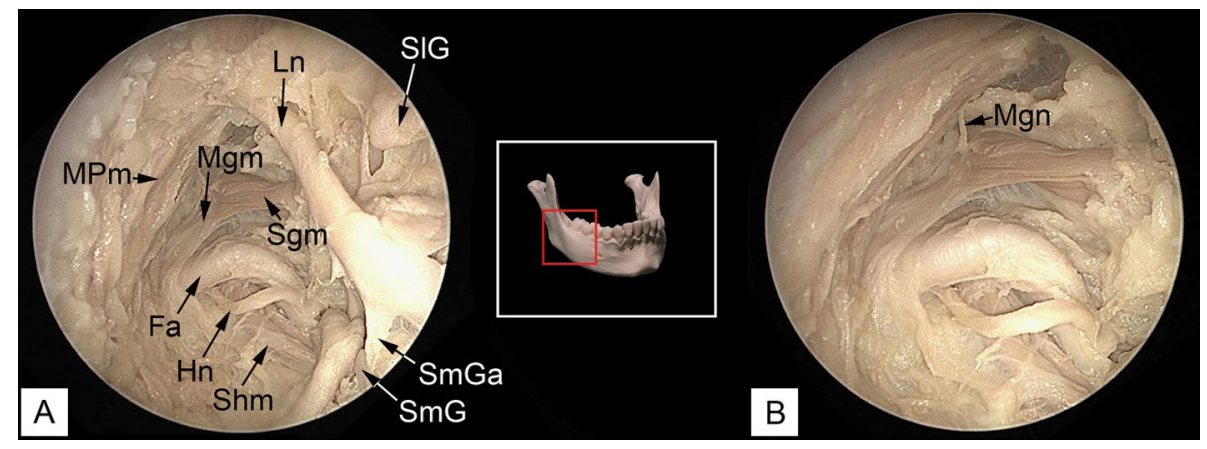

Figure 6. Endoscopic view representing the main topographic relationship of myloglossus. Both pictures were taken with a zero degree endoscope positioned into the posterior sublingual region in an antero-lateral to postero-medial direction; A. Overview image; B. Innervating branch; Fa — facial artery; Hn — hypoglossus nerve; Ln — lingual nerve; Mgm — myloglossus muscle; Mgn — myloglossal nerve; MPm — medial pterygoid muscle; Sgm — styloglossus muscle; Shm — stylohyoid muscle; SIG — sublingual gland (tail); SmG — submandibular gland; SmGa — submandibular ganglion. 
identified, with a significant association between muscular appearance and origin from the medial pterygoid muscle, whereas in MGL or mixed cases $100 \%$ of bony insertion was found.

About distal insertion, it was described as "the side of the base of the tongue" [3], "the side of the tongue" [19], the mass of lingual muscles between palatoglossal and styloglossal insertions [8] or "the fibres of the tongue" [13]. Here we observed a significant association between MGM and lingual insertion, whereas the MGL or mixed structure joined the SGM in all cases.

Furthermore, as regards the fibre direction, the posterolateral-to-anteromedial trajectory of the MGM together with the aforementioned findings supports its possible role in moving posteriorly the tongue. In particular, similarly to SGM, MGM could be involved both in the last phases of deglutition [14, 17], by creating the way for bolus passage and pushing it towards the oropharynx, and mastication, by avoiding tongue chewing with a rhythmic tongue retraction. Moreover, MGL could serve as passive anchorage for SGM, thus supporting its function.

Vascularisation. The facial artery was mostly found to pass either caudally or cranially to the myloglossal structure before reaching the submandibular gland. It usually released one or more branches where it turns downwards to reach the submandibular gland. When the proximal insertion was very anterior, the feeding branches arose from the loop around the submandibular gland; otherwise, the feeding artery(ies) could arise from the lingual artery and run caudo-cranially towards the myloglossus. Interestingly, common vascularisation with the medial pterygoid muscle (pattern B and C) was provided only by the facial artery, whereas common vascularisation with SGM (pattern D) and isolated vascularisation (pattern A) arose from both facial and lingual artery. To date, other data about the vascularisation are not present in literature.

Innervation. Several nervous branches were found reaching myloglossal structure. Coming from the buccal nerve, the innervating branch arose anterolaterally to the lateral pterygoid muscle and descended towards the superior surface of the myloglossus. Arising from the mylohyoid nerve, the innervating branch ascended to the inferior surface of the myloglossus posterior to the mylohyoid muscle. Likewise, when a hypoglossal branch was found, the nerve reached the muscle from below. Similar double in- nervation was previously reported by Nakajima and Nakamura [13], who described the trigeminal branch coming from tensor veli palatini nerve.

Explanation of these patterns of innervation could be given considering different points of view. About buccal nerve, innervation from this nerve alone, which is supposed to be mostly a sensory branch, suggests the presence of motor fibre within it; this should be explained as: 1) the buccal nerve arise from a common trunk with the anterior deep temporal nerve for the temporalis muscle innervation [6] and give branches for the lateral pterygoid muscle [7]; 2) the buccal nerve connects with the buccal branch of the facial nerve $[15,17]$. About additional innervation from hypoglossal nerve, it could have different interpretations: 1) the trigeminal branches might provide proprioception of the muscle and the hypoglossal nerve might provide motor innervation; 2 ) all nerves could provide motor innervation; 3 ) one nerve could not be functional, thus representing a rudimental remnant.

In addition, it is noteworthy to underline some embryological aspects linked to the myloglossal structure. During embryogenesis, the mesoderm that will differentiate into muscular tissue could variably localize either on the medial surface of the mandible or on the styloid process (or both) due to their closeness in the embryo [1]. Therefore, some muscle bundles of the medial pterygoid muscle could fuse with the inferolateral portion of the SGM, thus forming a muscle (namely the MGM) lying midway with respect to the mentioned muscles. On contrary, when the two muscles keep slightly separate during embryogenesis, a ligamentous structure going from the mandible to the SGM could develop (i.e. MGL), sometimes housing rudimental muscle fibres as clue of its origin. This hypothesis is coherent with the findings of a number of Authors describing muscular or ligamentous fibres going from the mandible to SGM and/or the tongue [5, 9-11]. Moreover, the identification of "mixed appearance", double nerve supply, and statistical relationships between insertions and ipsilateral SGM size further support the abovementioned embryogenic mechanism.

\section{CONCLUSIONS}

In conclusion, according to our findings, the myloglossus should not be considered a rare anatomical variant. Its function and embryogenesis are far to be well known; so, additional radiological study could be of some use to increase the number of cases and therefore assess the prevalence of this muscle and quantify the relationship with other lingual muscles. 
Finally, the embryogenesis of this muscle seems to be a complex phenomenon and additional embryological and histological studies could be useful for its complete understanding.

\section{Acknowledgements}

The Authors thank the donors of the cadavers for their indispensable contribution to this anatomical study. The Authors thank Alberto Paderno, MD, Department of Otorhinology, Head and Neck Surgery, University of Brescia, Brescia (Italy) for its precious suggestions.

\section{REFERENCES}

1. Barnwell YM. The morphology of musculus styloglossus in fifteen-week human fetuses. Int J Oral Myol. 1977; 3(2): 8-46, indexed in Pubmed: 275225.

2. Bonamy C, Broca P, Beau ME (1866) Atlas d'anatomie descriptive du corps humain. Masson, Paris.

3. Dunglison R. A new dictionary of medical sciences. Charles Bowen, Boston, 1833.

4. Gruber W. Ueber den musculeus myloglossus bei mangel und vorkommen des M. styloglossus. Arch Pathol Anat Physiol Klin Med. 1980; 81: 453-457.

5. Hallett $\mathrm{CH}$. An account of the varieties of the muscular system met with in the dissecting room of the University of Edinburgh during the winter session 1847-1848. Edinb Med Surg J. 1849; 22: 1-6.

6. Katori Y, Yamamoto M, Asakawa S, et al. Fetal developmental change in topographical relationship between the human lateral pterygoid muscle and buccal nerve. J Anat. 2012; 220(4): 384-395, doi: 10.1111/j.1469-7580.2012.01478.x, indexed in Pubmed: 22352373.

7. Kim HJ, Kwak HH, Hu KS, et al. Topographic anatomy of the mandibular nerve branches distributed on the two heads of the lateral pterygoid. Int J Oral Maxillofac Surg. 2003; 32(4): 408-413, doi: 10.1054/ijom.2002.0382, indexed in Pubmed: 14505626.

8. Lang J, Jüde HD. [Anatomy of the retromolar region of the human mandible]. Anat Anz. 1973; 133(3): 276-282, doi: 10.14219/jada.archive.1953.0195, indexed in Pubmed: 4741508.

9. Macalister A. The Varieties of the Styloid Muscles. J Anat Physiol. 1870; 5(Pt 1): 28-31, indexed in Pubmed: 17230882.

10. Macalister A. Additional observations on muscular anomalies in human anatomy (third series) with a catalogue of the principal muscular variations hitherto published. Trans R Irish Acad, Dublin, 1875.

11. Mérida-Velasco JR, Rodríguez-Vazquez JF, de la Cuadra Blanco $C$, et al. Origin of the styloglossus muscle in the human fetus. J Anat. 2006; 208(5): 649-653, doi: 10.1111/j.1469-7580.2006.00570.x, indexed in Pubmed: 16637887.

12. Micheli-Pellegrini V. About muscle insertions in man (Proposal for a new nomenclature of striated muscle). Acta Otorhinolaryngol Ital. 2011; 31(3): 167-176, doi: 10.1159/isbn.978-3-318-01729-8, indexed in Pubmed: 22058593.

13. Nakajima K, Nakamura M. Rare case of myloglossus in Japanese cadaver: anatomical and developmental considerations. Anat Sci Int. 2008; 83(1): 1-5, doi: 10.1111/j.1447-073X.2007.00181.x, indexed in Pubmed: 18402081.

14. Rodella LF, Buffoli $B$, Boninsegna $R$, et al. The myloglossus muscle: anatomical and clinical observations. Italian J Anat Embryol. 2011; 116: 154.

15. Rodella LF, Buffoli B, Labanca $M$, et al. A review of the mandibular and maxillary nerve supplies and their clinical relevance. Arch Oral Biol. 2012; 57(4): 323-334, doi: 10.1016/j.archoralbio.2011.09.007, indexed in Pubmed: 21996489.

16. Rood SR, Langdon H, Klueber K, et al. Muscular anatomy of the tonsil and tonsillar bed: a reexamination. Otolaryngol Head Neck Surg. 1979; 87(4): 401-408, doi: 10.1177/019 459987908700402, indexed in Pubmed: 503501.

17. Standring S. Gray's anatomy. 40th Ed. Elsevier, London, 2009.

18. Valenti G. Sur un muscle mandibulo-glosse (M. myloglossus Wood). Arch Ital Biol. 1926; 75: 77.

19. Wood J. Variations in Human Myology Observed during the Winter Session of 1866-67 at King's College, London. Proceedings of the Royal Society of London. 1866; 15(0): 518-546, doi: 10.1098/rspl.1866.0119. 